\title{
Neurophysiology of swallowing:
}

\section{Effects of age and bolus type}

\author{
lanessa A. Humbert ${ }^{*}$, Michelle E. Fitzgerald, Donald G. McLaren, Sterling Johnson, Eva \\ Porcaro, Kris Kosmatka, Jacqueline Hind, and JoAnne Robbins \\ William S. Middleton Memorial Veterans Hospital, Geriatric Research Education and Clinical Center, \\ Madison, WI, USA University of Wisconsin, Madison, USA
}

\begin{abstract}
This study examined age-related changes in swallowing from an integrated biomechanical and functional imaging perspective in order to more comprehensively characterize changes in swallowing associated with age. We examined swallowing-related fMRI brain activity and videoflouroscopic biomechanics of three bolus types (saliva, water and barium) in 12 young and 11 older adults. We found that age-related neurophysiological changes in swallowing are evident. The group of older adults recruited more cortical regions than young adults, including the pericentral gyri and inferior frontal gyrus pars opercularis and pars triangularis (primarily right-sided). Saliva swallows elicited significantly higher BOLD responses in regions important for swallowing compared to water and barium. In separate videofluoroscopy sessions, we obtained durational measures of supine swallowing. The older cohort had significantly longer delays before the onset of the pharyngeal swallow response and increased residue of ingested material in the pharynx. These findings suggest that older adults without neurological insult elicit more cortical involvement to complete the same swallowing tasks as younger adults.
\end{abstract}

\section{Introduction}

Swallowing is a complex phenomenon that involves tightly coupled interdependence among ongoing sensory and motor events before food enters the oral cavity until the swallow is complete. Dysphagia (swallowing difficulty) often leads to aspiration (ingested material entering the trachea), and may result from impairment of one or more of the many sensorymotor events that comprise normal swallowing. The oral cavity, pharynx, and larynx contain some of the richest and most diverse sensory receptors of the body, represented by dense intricate nerve supply to these regions (Mu and Sanders, 2000). Exact timing for movement of structures important for swallowing (i.e. tongue, larynx, pharynx) is imperative and highly sensory-reliant, such that even a one-second delay in movement can result in airway invasion of ingested material (Perlman et al., 1994).

For many years, the medical literature has asserted that swallowing is controlled primarily by the brainstem (Doty, 1968; Jean, 2001). However, more recent research has provided evidence that cortical and subcortical structures play a critical role in swallowing control, showing consistent activity in the pre- and post-central gyri, as well as the anterior cingulate and insular cortices (Hamdy et al., 1999; Kern et al., 2001; Martin et al., 2004; Mosier and Bereznaya, 2001).

\footnotetext{
(c) 2008 Elsevier Inc. All rights reserved.

*Corresponding author. 98 N. Broadway, Suite 413, Baltimore, MD 21231, USA. Fax: +1 410502 3546. E-mail address: ihumber1@jhmi.edu (I.A. Humbert).
} 
An estimated 18 million adults have dysphagia in the United States alone (ECRI, 1999), with increased prevalence in advancing age due to higher rates of dysphagia-inducing neurological damage or disorders such as stroke (Robbins et al., 1993), Alzheimer's disease (Priefer and Robbins, 1997), and Parkinson's disease (Robbins et al., 1986). Almost $40 \%$ of healthy, independent-living seniors have reported dysphagia in their lifetime (Roy et al., 2007). Within healthy aging, swallowing kinematics (i.e. elevation of the larynx) are generally slower compared to younger adults, leaving more residue within the oral and pharyngeal cavities (Robbins et al., 1992; Tracy et al., 1989).

Age-related differences in sensory-motor function occur alongside changes in peripheral and central nerve conduction (Dorfman and Bosley, 1979). Previous functional Magnetic Resonance Imaging (fMRI) studies have reported both decreased and increased BloodOxygen-Level-Dependent (BOLD) signal in healthy older adults compared to young adults for motor tasks (D'Esposito et al., 1999; Heuninckx et al., 2005). Common sites of increased signal are the preand post-central gyri, supplementary motor area, insula, and inferior frontal gyrus pars opercularis for older adults (Heuninckx et al., 2005; Mattay et al., 2002). Age-related increases in BOLD response may be distinguished by complexity of the motor-task (Ward and Frackowiak, 2003); simple tasks revealed over-activation in motor networks (Mattay et al., 2002) and complex tasks resulted in over-activation in sensory and cognitive cortical regions (Heuninckx et al., 2005).

Given the known kinematic changes in swallowing with healthy aging and age-related increased risk for dysphagia, a prospective comparison of swallowing neurophysiology between young and old adults is needed. Establishing a clear understanding of healthy swallowing neurophysiology in older adults will facilitate differentiation among ageappropriate normalcy, at-risk indicators of aging, and neurogenic dysphagia. The primary goal of this study was to prospectively compare neural activation in regions known to be important for swallowing between healthy old and young adults. We hypothesized that older participants would elicit BOLD response in more cortical regions within the swallowing network identified by previous research and in larger clusters than the younger participants. Since swallowing kinematics change with varied sensory input (i.e. taste, texture) (Ding et al., 2003), we examined differences in swallow types among sweetened barium, water and saliva. We hypothesized that barium would elicit more regions of activation and have higher BOLD signal in the ventrolateral postcentral gyrus (primary somatosensory cortex for the face, pharynx and larynx).

\section{Material and methods}

\section{Participants}

Twenty-three healthy, non-dysphagic participants completed the study (12 young: mean age 27.9, range 23-37, SD 4 yrs, 6 female; 11 old: mean age 72.3, range 64-83; SD 7.5, 6 female). No individual participated if he or she had a swallowing, speech or other otolaryngologic disorder, chronic medical condition, neurological or psychiatric disorder, or contraindications to being in the MRI environment. Three participants were left-handed ( 2 young females, 1 old male) as determined by the Edinburgh Handedness Inventory (Raczkowski et al., 1974). All participants provided written informed consent, which was approved by the Institutional Review Board of the University of Wisconsin.

\section{fMRI procedures}

Prior to the experimental procedures, a brief training session familiarized each participant with swallowing in the supine position and to the feel, taste and texture of the various boluses. The experimental tasks involved 50 swallows each in $5 \mathrm{ml}$ amounts (20 barium, 20 water, 10 saliva) 
presented in pseudo-random order and evenly distributed among three 5-minute runs. Barium (Varibar® Thin, E-Z-EM Inc) is a contrast material that is commonly used during clinical radiographic diagnosis of swallowing using videofluoroscopy. Water and barium (thin-liquid) were infused directly into the oral cavity via plastic tubing that was dispensed by a MR-safe injector (Spectris Solaris ${ }^{\circledR}$, Medrad) over a 2 -second period to avoid squirting material into the posterior pharynx (Fig. 1A). For the barium and water swallows, participants were instructed to swallow once they felt that the liquid had completely entered their mouths and to avoid multiple swallows. Saliva swallows were cued visually by the message "swallow saliva once". Non-swallow intervals were visually cued "do not swallow" and interspersed among the swallowing tasks. Inter-stimulus intervals for barium and water boluses were each $14.5 \mathrm{~s}$ and saliva swallows and relax intervals were each $13 \mathrm{~s}$. Visual cues were presented through a mirror that was mounted atop the head coil that reflected a screen positioned at the foot of the scanning bed. Presentation software (www.neuro-bs.com) was used to deliver visual stimuli from a personal computer.

\section{Swallow monitoring}

Swallowing events (whether cued or not) were monitored while fMRI was ongoing with a water-filled tube that extended from each participant's mouth to a pressure transducer in the control room (ADInstruments, Colorado Springs CO) (Fig. 1A). Pressure changes in the oral cavity that occurred during swallowing displaced the water in the tube, providing the data acquisition system with a signal differential from rest (Fig. 1B). These oral pressure changes were monitored to ensure task compliance and to identify inadvertent swallows and were triggered by and synchronized with: (1) the onset of the transistor-transistor logic (TTL) pulse derived from the MRI scan acquisition, and (2) the personal computer used to present the visual stimuli so that swallow onsets, visual cues, and the MRI pulses were time-locked. Thus, our data acquisition equipment (PowerLab 16/30, ADInstruments) obtained real-time oral pressure signals, stimulus onset times and the TTL pulse for the duration of each experimental run.

\section{Functional MRI}

Imaging protocol-Magnetic resonance images were collected on a 3.0 T GE Signa scanner (Waukesha, WI) using an 8-channel head coil. Functional image data were obtained with a $\mathrm{T} 2 *$ gradient-echo, echo planar imaging (EPI) pulse sequence optimized for blood-oxygen level dependent $(\mathrm{BOLD})$ contrast with the following parameters: echo time $(\mathrm{TE})=30 \mathrm{~ms}$; repetition time $(\mathrm{TR})=2000 \mathrm{~ms}$; flip angle $=75^{\circ}$; acquisition matrix $=64 \times 64$; and FOV $=240$ $\mathrm{mm}$. Whole brain coverage was provided with thirty $4.5 \mathrm{~mm}$ thick inter-leaved axial slices. In each of the three runs, 148 volumes were collected and the first three discarded to allow for magnetization stabilization. Higher order shimming was applied to the static magnetic field (B0) prior to EPI acquisition.

Additionally, a whole-brain high-resolution T1-weighted Inversion-Recovery 3D spoiled gradient echo (3D-IRSPGR) was collected with the following parameters: $\mathrm{TR}=9.592 \mathrm{~ms}$; TE $=3.00 \mathrm{~ms}$; inversion time $(\mathrm{TI})=600 \mathrm{~ms}$; flip angle $=10^{\circ}$; acquisition matrix $=256 \times 256$; FOV $=230 \mathrm{~mm}$; slice thickness $=1.5 \mathrm{~mm}$. The T1-weighted images were inspected by a neuroradiologist to confirm normal brain anatomy and were used to investigate structural differences between groups.

fMRI image processing-All functional images were processed with an automated script using components of the Statistical Parametric Mapping (SPM5, Wellcome Department of Imaging Neuroscience, University College London, UK) and Analysis of Functional NeuroImages (AFNI, Medical College of Wisconsin, USA) software packages. Images were slice-timing corrected, motion-corrected, normalized to the MNI EPI template using a nonlinear transform, re-sampled to $2 \mathrm{~mm}$ isotropic voxels, and smoothed with $8 \mathrm{~mm}$ FWHM 
Gaussian kernel. All individuals had less than $3 \mathrm{~mm}$ of movement in the $x, y$, and $z$ directions and less than $3^{\circ}$ of deflection in pitch, roll, and yaw.

fMRI statistics-First-level analyses of the time series data were performed for individual participants using a general linear model. Rather than using stimulus presentation times, swallow onset times for each condition (barium, water, and saliva) were obtained directly using the intra-oral pressure-monitoring device described above and in Fig. 1. A separate reference function was made for each subject based on individual delay periods. Swallow response delays (time between swallow stimulus and swallow onset) averaged $2.3 \mathrm{~s}$ for all swallows (means: saliva 0.5; water 3.21; barium 3.07; old participant mean delay $2.3 \mathrm{~s}$, young mean delay $2.1 \mathrm{~s}$ ). In some cases, participants swallowed during the inter-stimulus intervals creating a fourth swallow condition (spontaneous). This fourth condition was included in the design matrix when applicable, but not considered further. The vectors of onset for each condition were convolved with the canonical hemodynamic response function (HRF) to construct the statistical model, resulting in a 3 or 4 column design matrix (depending on presence of the spontaneous condition). The general linear-model removed the low frequencies with a $128 \mathrm{~s} \mathrm{high-pass} \mathrm{filter.}$ Additionally, vectors were added to the design matrix for the motion parameters and their backward derivatives. In general, correlation of intra-scan motion with the functional task can confound statistical inference in the model (Johnstone et al., 2006). In order to verify the significance of task correlated motion in this study, a Pearson Correlation test was calculated between post-swallow-onset vectors (1 TR offset) and: (1) each of the six realignment parameter time series (a measure of displacement), as well as; (2) the backwards derivative of each realignment parameter time series (an estimate of velocity). The results indicated a moderate magnitude of task correlation for all of the motion parameters and their derivatives. These results confirm the need to add them as regressors in the model. Further, $t$-tests (MannWhitney) on the correlation r-values revealed no significant difference in task-correlated motion between groups $(p>0.05)$.

Second-level analyses for age and swallow type differences used repeated measures $2 \times 3$ ANOVAs with BOLD amplitude images for barium, water, and saliva for each participant as the dependent measures. BOLD amplitude images were from first-level contrasts of each swallow type. First, we used a repeated measures $2 \times 3$ ANOVA that included terms for subject, age group, swallow type and age and swallow type interactions to determine regions where there was an interaction between group and swallow type; regions that contained statistically significant interactions are reported and then excluded from subsequent analyses of main effects of swallow type and group. Main effects ( $F$-test) for age group and swallow type were computed from a repeated measures $2 \times 3$ ANOVA that include terms for subject, age, and swallow type. Subsequently, $T$-contrasts were used to determine significant differences between water, barium and saliva swallows and significant age differences within regions showing a main effect. Additionally, our analysis was further restricted to the following cortical regions that are known to be involved in swallowing: precentral gyrus, postcentral gyrus, insular cortex, anterior cingulate cortex, supplementary motor area, supramarginal gyrus, superior frontral gyrus, middle frontal gyrus, inferior frontal gyrus, superior parietal gyrus, middle parietal gyrus, inferior parietal gyrus, frontal inferior opercularis, frontal inferior triangularis, and the Rolandic operculum. All analyses were at the $p=0.001$ uncorrected threshold level in at least 20 edge-connected voxels. A one-sample $T$-test was used to individually compare the amount of BOLD response of each swallow stimulus type to zero within the bilateral ventrolateral postcentral gyri (significance set at Bonferroni corrected alpha level of $p \leq 0.016$ ).

To determine whether age-related atrophy of cortical grey matter overlapped with regions where there were significant group differences in BOLD signal during swallowing activity, posterior probability maps for grey matter were computed using the VBM5 toolbox 
(http://dbm.neuro.uni-jena.de/vbm/) in SPM5 and entered into a two-sample T-test to compare the age groups. Results were constrained to regions known to be involved in swallowing.

\section{Videofluoroscopic procedures}

Swallowing biomechanics were obtained to confirm that physiological differences exist between the 2 age groups in this study. Furthermore, since lingual and pharyngeal kinematics differ in supine compared to upright swallowing (Dejaeger et al., 1994), biomechanics from supine swallowing were needed to ensure similarity with the fMRI environment where subjects also lay supinely. Videofluoroscopy is a radiographic technique that provides information on swallowing including biomechanics of the upper aerodigestive tract and bolus flow. Full resolution fluoroscopic images were captured in real time (30 frames per second) and digitally recorded for analysis (Siemens Sireskop). The image intensifier was focused on the oral cavity, the posterior pharyngeal wall, and just below the upper esophageal sphincter in the lateral plane. Two speech-language pathologists and a radiologist conducted all videofluoroscopic swallowing studies prior to fMRI procedures.

All participants swallowed three- $5 \mathrm{ml}$ thin liquid barium boluses, two $5 \mathrm{ml}$-water boluses and one saliva swallow in random order. The barium and water boluses were manually dispensed onto the mid tongue with a syringe and plastic tubing (Medrad® model SSIT_96VLD) as in fMRI procedures.

Videofluoroscopic image analysis-Group differences in durational measures of supine swallowing biomechanics (i.e. hyolaryngeal excursion, UES opening) and bolus flow were obtained by using standard criteria and definitions (Lof and Robbins, 1990; Robbins et al., 1992). For barium swallows, post-swallow barium contrast residue was judged from the videofluoroscopic image when the hyoid bone returned to rest, operationally defining the end of the swallow. Measurements were taken in the oral cavity, vallecula, posterior pharyngeal wall, pyriform sinus, and upper esophageal sphincter. Ratings were scaled on a 3-point system, in which 0 corresponded to no barium residue, 1 to a coating of barium residue (a line of barium on a structure), and 2 to pooling of barium (an area larger than a line of barium on a structure). Interjudge reliability of $84 \%$ and an intrajudge reliability of $90 \%$ agreement previously have been reported by using similar datasets (Hind et al., 2001). The 8-point Penetration-Aspiration Scale (Robbins et al., 1999; Rosenbek et al., 1996) was used to score each swallow observed during the videofluoroscopic swallowing evaluation. Scores on this scale reflect the occurrence, anatomic depth, subject response to, and clearance of material invading the laryngeal vestibule or trachea. All swallowing judges were blinded to age group.

Videofluoroscopy statistics-The main effects of age on supine swallowing biomechanics, residue and swallowing severity were determined using the Mann-Whitney Test and differences in bolus stimuli (barium, water, saliva) were determined using Friedman's Test for repeated measures (SPSS 16.0 for Mac). Intra-class correlation coefficients (ICC) were computed for all biomechanical measures. The ICC represents the proportion of total variation (between-subject variability and measurement variability) that may be attributed to betweensubject variability. Values near 1 suggest nearly all variability is essentially biological variance and not related to measurement, whereas values near 0 indicate that variability is primarily a result of measurement problems (Fleiss, 1999).

\section{Results}

No signs of aspiration were observed during the videofluoroscopic or fMRI procedures. Overall, task compliance was $99 \%$ for swallowing during fMRI procedures as determined by the intra-oral pressure device output. There were no statistically significant differences in head 
movement between groups. Overlap between significant differences in grey matter atrophy and active areas for swallowing between groups were found in no more than 80 voxels, suggesting that atrophy is not confounding the results.

\section{fMRI results}

Age by swallow type interaction-The left anterior superior frontal gyrus (MNI - 2644 46; 54-voxel cluster) was significant for an interaction for age and swallow type.

Main effect of age (Fig. 2, Table 1)—The main effects of age revealed significant differences in BOLD signal in the bilateral ventrolateral pre- and post-central gyri, bilateral frontal lobe (inferior, middle and superior gyri), bilateral parietal regions (inferior and superior gyri) and the right superior temporal gyrus (Fig. 2A and Table 1).

Age comparisons (Fig. 3, Table 2)-To further describe the direction and location of effects by age-group, these age-comparison contrasts were derived from the data obtained in the main effects of age. The BOLD signal from the older participants is largely driving the response found in the main effects of age. For all swallows, the older cohort showed significantly higher BOLD response than the younger group across a large region of the cortical swallowing network, involving the right pre- and post-central gyri, bilateral frontal lobe (inferior, middle and superior gyri), bilateral parietal regions (inferior and superior gyri) and the right superior temporal gyrus. Younger participants showed higher BOLD response in the left pre- and post-central gyri, left supplementary motor area (SMA), and right superior frontal gyrus (Fig. 3, Table 2).

Within the older group, BOLD signal in the ventro-lateral post-central gyrus was highest in the right hemisphere for all swallow stimulus types, but the younger group had higher BOLD signal in the left hemisphere for the same region (Fig. 2C). Statistically significant differences were found between age groups for saliva swallow activation in the left ventro-lateral postcentral gyrus ( $p=0.011)$ (Fig. 2C).

Main effect of swallow type (Fig. 4, Table 3)-The main effects of swallow stimulus types had significant BOLD signal the bilateral ventrolateral pre-central gyri, bilateral inferior frontal gyri (IFG) pars opercularis, and left IFG pars triangularis (Fig. 4, Table 3).

Swallow stimulus comparisons (Fig. 5, Table 4)-Swallow stimulus comparisons were derived from the main effects of swallow type and reveal that saliva swallows are largely responsible for the response for this main effect. Saliva swallows elicited more active cortical regions than water and barium overall. Higher BOLD responses to saliva than water were found in the bilateral IFG pars opercularis and right IFG pars triangularis, left precentral gyrus, bilateral SMA, left insula and middle frontal gyrus and right anterior cingulate cortex. Higher BOLD responses to saliva than to barium were found in the left IFG pars opercularis, left precentral gyrus, left Rolandic operculum, right SMA, and bilateral inferior parietal gyri, left superior and middle frontal gyri, and right middle cingulate cortex. The only other significant contrast of swallow type was water>saliva, where the right, insula/IFG pars opercularis region was most active.

Within the ventrolateral postcentral gyrus, saliva swallows elicited the highest BOLD response bilaterally of the 3 swallow types $(p \leq 0.001)$, showing saliva swallows are driving this effect (Fig. 5, Table 4).

Activation within the IFG pars opercularis, which was a part of the largest cluster with the strongest activation for the main effects of swallow, was greatest for saliva swallows for both age groups (Fig. 4C). 


\section{Supine swallow biomechanical results}

Duration-Stage transition duration (STD) represents the time between the head of the bolus reaching the ramus of the mandible until the hyoid bone begins elevation. The older participants had significantly longer STD measures than the younger cohort ( $p<0.001 ;$ ICC 0.089$)$. No other swallowing biomechanical comparison survived the corrected alpha threshold level of $p=$ 0.016 .

Residue-Older participants had significantly more residue in the valecullae $(p<0.037)$ and upper esophageal sphincter $(p<0.035)$ than the young cohort after swallowing in the supine position.

Penetration aspiration scale-No statistically significant difference was found between these healthy age groups for the Penetration-Aspiration scale.

\section{Discussion}

Age

This is the first prospectively designed study to compare both the neural correlates of swallowing between young and old adults as well as the relationship of activation to the stimulus swallowed. Results indicate that young and old participants demonstrated significantly different neural responses to the same swallowing tasks. As hypothesized, healthy older participants had activity in more regions of the cortex than the young for swallowing. The older adults may have shown more neural activation for swallowing because they required more effort to swallow compared to the younger cohort. Similar effort-related increases in cortical activation were reported for voluntary sphincteric contractions at maximal versus submaximal intensities (Kern et al., 2004)and in memory tasks that require varying levels of effort (Jansma et al., 2007). Other studies have shown age-related increases in neural activation during limb movement in the old compared to the young within certain brain regions (Heuninckx et al., 2005; Hutchinson et al., 2002). Thus, age-related differences in peripheral movement (i.e. slower swallows in older individuals) (Robbins et al., 1992) may occur along side differences in neural control of swallowing. However, increased neural activation as a consequence of swallowing effort was not directly examined in this investigation. Therefore, an alternative explanation for increased neural activation within the older group could be that younger participants are simply more efficient in their cortical use for the same task with equal effort than are older adults.

The older group had more active voxels above the designated threshold level than the young group on the right side (right 7768 voxels; left 1492 voxels), whereas the young>old comparison showed more left-sided active voxels (right 65 voxels; left 362 voxels). Previous studies have shown right-hemispheric dominance for attention in healthy individuals (Heilman and Van Den Abell, 1980; Verfaellie et al., 1988) and that post-stroke patients with right hemispheric damage have difficulty with sustained attention (Saldert and Ahlsen, 2007). The fMRI tasks in this study may have placed additional demands for attention on the older adults than the younger adults, eliciting more BOLD response in the right hemisphere. Additionally, the old $\mathrm{N}$ young comparison revealed more superior and middle frontal lobe activity than younger adults; these cortical regions are active during attention-demanding tasks (Butti et al., 2006; Lewin et al., 1996).

Only one known neuroimaging study using fMRI examined swallowing in healthy older adults and reported similar regions of activity, including the bilateral sensory-motor cortices, insula/ operculum, and cingulate cortex for saliva and water swallows (Martin et al., 2006). Although Martin et al. (2006) did not include young adults for a prospective comparison, two separate, 
previously conducted studies of young (Martin et al., 2004)and old(Martin et al., 2006) healthy adults have shown strong left-hemisphere lateralization for saliva and water swallows. Unlike volitional limb movement, swallowing inherently recruits bilateral musculature to safely guide the bolus past the airway and into the esophagus. Therefore, it is difficult to identify, with certainty, swallowing hemispheric dominance for head and neck sensation and movement given the tasks from this study. Yet, unilateral paralysis (Ollivere et al., 2006) or paresis (Vigderman et al., 1998) of head or neck musculature can result in serious swallowing disability, as can unilateral cortical damage secondary to stroke (Daniels and Foundas, 1997; Robbins et al., 1993). The right-sided activity in the older group of the current study might indicate a normal shift toward right-hemispheric activity for swallowing with healthy aging. Therefore, dysphagia-inducing phenomena, such as stroke, warrant further investigation into the importance of laterality, if any, in normal swallowing across the age span.

\section{Swallow type}

We hypothesized that sweetened barium would recruit the most cortical regions and have higher BOLD responses in sensory areas because of the heightened sensory properties (taste and texture). In contrast to our hypothesis, saliva swallows activated a larger number of cortical regions that are associated Within diverse functions, including mainly the left ventrolateral aspect of the precentral gyrus, involved in motor execution (Penfield and Boldery, 1937), and IFG pars operculum, involved in speech, voice and swallowing motor planning (Hillis et al., 2004; Jurgens, 2002; Watanabe et al., 2004). Saliva swallows may have elicited more BOLD response because it is more effortful with minimal sensory stimulation in the oropharynx or when very small amounts are being swallowed. Saliva swallows become difficult to produce after numerous, sequential dry swallows where saliva is gradually diminished, involving more lingual pressure generation (Mansson and Sandberg, 1974). Also, numbing the oropharynx with topical anesthesia obliterates the pharyngeal swallow response (Mansson and Sandberg, 1975) and causes a pronounced decrease in motor cortex activation during swallowing (Teismann et al., 2007). On the other hand, water swallows had higher BOLD response only in the right insular-opercular region compared to saliva swallows. This result is supported by findings from an fMRI study where only lingual somatosensory stimuli specifically produced activation in the insula and surrounding opercular regions (including IFG) (Cerf-Ducastel et al., 2001). As well, Daniels et al. 1997 noted that patients with insular lesions demonstrated dysphagia characterized by delayed onset of the pharyngeal swallow. In the present study, water represents a primarily tactile stimulus to the oropharynx because it is tasteless (and at room temperature), whereas saliva provides very little stimulus and the barium was sweetened. However, saliva swallows elicited more activity in the postcentral gyrus than water swallows, likely because of the added lingual contact and somatosensory stimulation within the oral cavity and posterior wall when generating enough stimulus to produce a saliva swallow on command.

In contrast to our findings, Martin et al. (2006) found that water swallows activated four times more volume than saliva swallows in healthy older adults who were similar in age $(74.2 \pm 8.1$ years) to the older participants in this study. However, our water/saliva contrasts included both young and old participants, which might account for differences in BOLD signal and suggest that age-difference is driving our results. Noteworthy differences in the duration of oral pressure changes for saliva swallows were frequently observed in the output of the oral pressure-monitoring device (Fig. 6) between age groups. As well, older participants reported effortful periods of "getting the dry swallows going" during fMRI. Analogously, any increased lingual pumping generated by the older group could be comparable to fMRI studies where finger-tapping rate (a measure of task demand) affected the percent of active voxels in a given region of interest (Newton et al., 2007). 


\section{Swallowing biomechanics}

Consistent with the literature, swallowing biomechanics were found in the current study to differ between age groups. The older cohort had much longer delays in the onset of the pharyngeal response mechanism than the young. When a bolus is swallowed, the tongue propels the bolus posteriorly into the pharynx and numerous, varied sensory receptors are stimulated along the way, triggering the pharyngeal swallow response (Miller, 2002). The properties of a bolus (i.e. volume, viscosity, temperature) are detected by oropharyngeal sensory receptors and are used to guide motor function for swallowing. Many older adults have muted oral sensation compared to young adults (Calhoun et al., 1992; Ostreicher and Hawk, 1982). This difference might contribute to the longer delays in initiation of the pharyngeal swallow (STD or stage transition duration) observed in this study and in others (Logemann et al., 2002). In addition, older participants had significantly more post-swallow residue in the valleculae and upper esophageal sphincter, further demonstrating differences in their ability to efficiently clear a bolus from the pharyngeal cavity in the supine position.

\section{Clinical implications}

Much of swallowing research has focused on understanding neurogenic dysphagia. Recent medical technological advances, such as fMRI, have catapulted the field into investigations of normal swallowing to enhance what is already known about swallowing neurophysiology, although primarily in young adults. Our results revealed that saliva swallows involve increased neural activation over water and barium swallows. This suggests that interventions that involve frequent saliva swallowing tasks might require more neural activation in a disordered population (Kaas et al., 1983), while maintaining safety in patients whose dysphagia is too severe for oral intake of fluids. Age comparisons point to the likelihood of more right-sided neural activity for swallowing with advancing age, leading to the possibility that right-sided brain damage (i.e. stroke, traumatic brain damage) in the old may result in more severe swallowing impairment, which is consistent with findings by Robbins et al. (1993).

Furthermore, sensory-reliant aspects of swallowing, such as initiating the swallowing response, may rely on very specific aspects of the primary sensory cortex, such as the ventrolateral postcentral gyrus, yet involve related sensory networks that might have been otherwise overlooked for the purposes swallowing function (i.e. orbital frontal gyrus). Based on the results from this study, it is likely that impaired sensory - motor swallowing abilities are reflected by differences in neural responses and guide diagnosis and treatment in neurogenic dysphagia.

\section{Limitations}

Some technical constraints limit our results, including: (1) limited swallowing trials with videofluoroscopy to minimize participant exposure to harmful radiation; (2) inability to image swallow biomechanics and the brain simultaneously; and, (3) stage transition duration can only be measured with barium swallow trials. Furthermore, swallowing tasks were completed in the supine position and cannot be directly generalized to swallowing biomechanics in an upright position. Saliva swallows were cued differently (visual) than water and barium swallows (tactile) to ensure that an adequate number of saliva swallows could be analyzed and so that they occurred at predictable intervals (which might account for some differences in activation). Finally, there is a possibility that saliva and water swallows that followed barium swallows may have included trace amounts of barium, possibly affecting BOLD response for those swallow types.

\section{Conclusions}

This investigation is the first reported prospective comparison of swallowing neurophysiology in healthy young and old adults and the first to examine the BOLD response to swallowing barium, which is so frequently utilized in dysphagia testing. Our findings indicate a difference 
in neural activation for swallowing between young and old healthy adults. Older adults recruit more cortical regions overall and more right-sided cortical activity for swallowing compared to younger adults. Saliva swallows activated more cortical regions and had a higher BOLD response bilaterally in the ventrolateral postcentral gyrus compared to water. These findings warrant further investigation into swallow-type and age-related differences in the neurophysiology of swallowing. Neurologically-based dysphagia may be better understood based on these initial findings.

\section{Acknowledgments}

This study was supported by the National Institutes of Health (NIH), NCRR, The Training and Education to Advance Multidisciplinary-Clinical-Research (TEAM) Program, 8K12RR023268-02.

National Institutes of Health and National Institute on Aging grant no. T32 AG20013 and the William S. Middleton Memorial VA Hospital Geriatric Research Education and Clinical Center (GRECC).

This is GRECC manuscript \#2008-16.

\section{References}

Butti M, Pastori A, Merzagora A, Zucca C, Bianchi A, Reni G, et al. Multimodal analysis of a sustained attention protocol: continuous performance test assessed with near infrared spectroscopy and EEG. Conf. Proc. IEEE Eng. Med. Biol. Soc 2006;1:1040-1043. [PubMed: 17946440]

Calhoun KH, Gibson B, Hartley L, Minton J, Hokanson JA. Age-related changes in oral sensation. Laryngoscope 1992;102:109-116. [PubMed: 1738279]

Cerf-Ducastel B, Van de Moortele PF, MacLeod P, Le Bihan D, Faurion A. Interaction of gustatory and lingual somatosensory perceptions at the cortical level in the human: a functional magnetic resonance imaging study. Chem. Senses 2001;26:371-383. [PubMed: 11369672]

Daniels SK, Foundas AL. The role of the insular cortex in dysphagia. Dysphagia 1997;12:146-156. [PubMed: 9190100]

Dejaeger E, Pelemans W, Ponette E, Vantrappen G. Effect of body position on deglutition. Dig. Dis. Sci 1994;39:762-765. [PubMed: 8149842]

D’Esposito M, Zarahn E, Aguirre GK, Rypma B. The effect of normal aging on the coupling of neural activity to the bold hemodynamic response. NeuroImage 1999;10:6-14. [PubMed: 10385577]

Ding R, Logemann JA, Larson CR, Rademaker AW. The effects of taste and consistency on swallow physiology in younger and older healthy individuals: a surface electromyographic study. J. Speech Lang. Hear. Res 2003;46:977-989. [PubMed: 12959474]

Dorfman LJ, Bosley TM. Age-related changes in peripheral and central nerve conduction in man. Neurology 1979;29:38-44. [PubMed: 570675]

Doty R. Neural organization of deglutition. Am Physiol Soc 1861-1902 Vol sect. VI 1968;IV, 1956. Washington, DC

ECRI E-BPC-. Diagnosis and Treatment of Swallowing Disorders (Dysphagia) in Acute Stroke Patients. Agency for Health Care Policy and Research; Rockville MD: 1999.

Fleiss, JL. The Design and Analysis of Clinical Experiments. John Wiley \& Sons, Inc; New York: 1999.

Hamdy S, Mikulis DJ, Crawley A, Xue S, Lau H, Henry S, et al. Cortical activation during human volitional swallowing: an event-related fMRI study. Am. J. Physiol 1999;277:G219-G225. [PubMed: 10409170]

Heilman KM, Van Den Abell T. Right hemisphere dominance for attention: the mechanism underlying hemispheric asymmetries of inattention (neglect). Neurology 1980;30:327-330. [PubMed: 7189037]

Heuninckx S, Wenderoth N, Debaere F, Peeters R, Swinnen SP. Neural basis of aging: the penetration of cognition into action control. J. Neurosci 2005;25:6787-6796. [PubMed: 16033888]

Hillis AE, Work M, Barker PB, Jacobs MA, Breese EL, Maurer K. Re-examining the brain regions crucial for orchestrating speech articulation. Brain 2004;127:1479-1487. [PubMed: 15090478] 
Hind JA, Nicosia MA, Roecker EB, Carnes ML, Robbins J. Comparison of effortful and noneffortful swallows in healthy middle-aged and older adults. Arch. Phys. Med. Rehabil 2001;82:1661-1665. [PubMed: 11733879]

Hutchinson S, Kobayashi M, Horkan CM, Pascual-Leone A, Alexander MP, Schlaug G. Age-related differences in movement representation. NeuroImage 2002;17:1720-1728. [PubMed: 12498746]

Jansma JM, Ramsey NF, de Zwart JA, van Gelderen P, Duyn JH. fMRI study of effort and information processing in a working memory task. Hum. Brain Mapp 2007;28:431-440. [PubMed: 17133397]

Jean A. Brain stem control of swallowing: neuronal network and cellular mechanisms. Physiol. Rev 2001;81:929-969. [PubMed: 11274347]

Johnstone T, Walsh Ores K.S. Greischar LL, Alexander AL, Fox AS, Davidson RJ, et al. Motion correction and the use of motion covariates in multiple-subject fMRI analysis. Hum. Brain Mapp 2006;27:779-788. [PubMed: 16456818]

Jurgens U. Neural pathways underlying vocal control. Neurosci. Biobehav. Rev 2002;26:235-258. [PubMed: 11856561]

Kaas JH, Merzenich MM, Killackey HP. The reorganization of somatosensory cortex following peripheral nerve damage in adult and developing mammals. Annu. Rev. Neurosci 1983;6:325-356. [PubMed: 6340591]

Kern MK, Jaradeh S, Arndorfer RC, Shaker R. Cerebral cortical representation of reflexive and volitional swallowing in humans. Am. J. Physiol. Gasterointest. Liver Physiol 2001;280:G354-G360.

Kern MK, Arndorfer RC, Hyde JS, Shaker R. Cerebral cortical representation of external anal sphincter contraction: effect of effort. Am. J. Physiol.: Gasterointest. Liver Physiol 2004;286:G304-G311.

Lewin JS, Friedman L, Wu D, Miller DA, Thompson LA, Klein SK, et al. Cortical localization of human sustained attention: detection with functional MR using a visual vigilance paradigm. J. Comput. Assist. Tomogr 1996;20:695-701. [PubMed: 8797896]

Lof GL, Robbins J. Test-retest variability in normal swallowing. Dysphagia 1990;4:236-242. [PubMed: 2209099]

Logemann JA, Pauloski BR, Rademaker AW, Kahrilas PJ. Oropharyngeal swallow in younger and older women: videofluoroscopic analysis. J. Speech Lang. Hear. Res 2002;45:434-445. [PubMed: 12068997]

Mansson I, Sandberg N. Effects of surface anesthesia on deglutition in man. Laryngoscope 1974;84:427437. [PubMed: 4814413]

Mansson I, Sandberg N. Oro-pharyngeal sensitivity and elicitation of swallowing in man. Acta Otolaryngol 1975;79:140-145. [PubMed: 1146533]

Martin RE, MacIntosh BJ, Smith RC, Barr AM, Stevens TK, Gati JS, et al. Cerebral areas processing swallowing and tongue movement are overlapping but distinct: a functional magnetic resonance imaging study. J. Neurophysiol 2004;92:2428-2443. [PubMed: 15163677]

Martin R, Barr A, Macintosh B, Smith R, Stevens T, Taves D, et al. Cerebral cortical processing of swallowing in older adults. Exp. Brain Res. 2006

Mattay VS, Fera F, Tessitore A, Hariri AR, Das S, Callicott JH, et al. Neurophysiological correlates of age-related changes in human motor function. Neurology 2002;58:630-635. [PubMed: 11865144]

Miller AJ. Oral and pharyngeal reflexes in the mammalian nervous system: their diverse range in complexity and the pivotal role of the tongue. Crit. Rev. Oral Biol. Med 2002;13:409-425. [PubMed: 12393760]

Mosier K, Bereznaya I. Parallel cortical networks for volitional control of swallowing in humans. Exp. Brain Res 2001;140:280-289. [PubMed: 11681303]

$\mathrm{Mu} \mathrm{L}$, Sanders I. Sensory nerve supply of the human oro- and laryngopharynx: a preliminary study. Anat. Rec 2000;258:406-420. [PubMed: 10737859]

Newton AT, Morgan VL, Gore JC. Task demand modulation of steady-state functional connectivity to primary motor cortex. Hum. Brain Mapp 2007;28:663-672. [PubMed: 17080441]

Ollivere B, Duce K, Rowlands G, Harrison P, O'Reilly BJ. Swallowing dysfunction in patients with unilateral vocal fold paralysis: aetiology and outcomes. J. Laryngol. Otol 2006;120:38-41. [PubMed: 16359143] 
Ostreicher HJ, Hawk AM. Patterns of performance for two age groups of normal adults on a test of oral form discrimination. J. Commun. Disord 1982;15:329-335. [PubMed: 7119143]

Penfield W, Boldery E. Somatic motor and sensory representation in the cerebral cortex of man as studied by electrical stimulation. Brain 1937;60:389-443.

Perlman AL, Booth BM, Grayhack JP. Videofluoroscopic predictors of aspiration in patients with oropharyngeal dysphagia. Dysphagia 1994;9:90-95. [PubMed: 8005013]

Priefer BA, Robbins J. Eating changes in mild-stage Alzheimer's disease: a pilot study. Dysphagia 1997;12:212-221. [PubMed: 9294942]

Raczkowski D, Kalat JW, Nebes R. Reliability and validity of some handedness questionnaire items. Neuropsychologia 1974;12:43-47. [PubMed: 4821188]

Robbins JA, Logemann JA, Kirshner HS. Swallowing and speech production in Parkinson's disease. Ann. Neurol 1986;19:283-287. [PubMed: 3963773]

Robbins J, Hamilton JW, Lof GL, Kempster GB. Oropharyngeal swallowing in normal adults of different ages. Gastroenterology 1992;103:823-829. [PubMed: 1499933]

Robbins J, Levine RL, Maser A, Rosenbek JC, Kempster GB. Swallowing after unilateral stroke of the cerebral cortex. Arch. Phys. Med. Rehabil 1993;74:1295-1300. [PubMed: 8259895]

Robbins J, Coyle J, Rosenbek J, Roecker E, Wood J. Differentiation of normal and abnormal airway protection during swallowing using the penetration- aspiration scale. Dysphagia 1999;14:228-232. [PubMed: 10467048]

Rosenbek JC, Robbins JA, Roecker EB, Coyle JL, Wood JL. A penetration-aspiration scale. Dysphagia 1996;11:93-98. [PubMed: 8721066]

Roy N, Stemple J, Merrill RM, Thomas L. Dysphagia in the elderly: preliminary evidence of prevalence, risk factors, and socioemotional effects. Ann. Otol. Rhinol. Laryngol 2007;116:858-865. [PubMed: 18074673]

Saldert C, Ahlsen E. Inference in right hemisphere damaged individuals' comprehension: the role of sustained attention. Clin. Linguist. Phon 2007;21:637-655. [PubMed: 17653972]

Teismann IK, Steinstraeter O, Stoeckigt K, Suntrup S, Wollbrink A, Pantev C, et al. Functional oropharyngeal sensory disruption interferes with the cortical control of swallowing. BMC Neurosci 2007;8:62. [PubMed: 17678546]

Tracy JF, Logemann JA, Kahrilas PJ, Jacob P, Kobara M, Krugler C. Preliminary observations on the effects of age on oropharyngeal deglutition. Dysphagia 1989;4:90-94. [PubMed: 2640185]

Verfaellie M, Bowers D, Heilman KM. Hemispheric asymmetries in mediating intention, but not selective attention. Neuropsychologia 1988;26:521-531. [PubMed: 3405398]

Vigderman AM, Chavin JM, Kososky C, Tahmoush AJ. Aphagia due to pharyngeal constrictor paresis from acute lateral medullary infarction. J. Neurol. Sci 1998;155:208-210. [PubMed: 9562269]

Ward NS, Frackowiak RS. Age-related changes in the neural correlates of motor performance. Brain 2003;126:873-888. [PubMed: 12615645]

Watanabe Y, Abe S, Ishikawa T, Yamada Y, Yamane GY. Cortical regulation during the early stage of initiation of voluntary swallowing in humans. Dysphagia 2004;19:100-108. [PubMed: 15382798] 

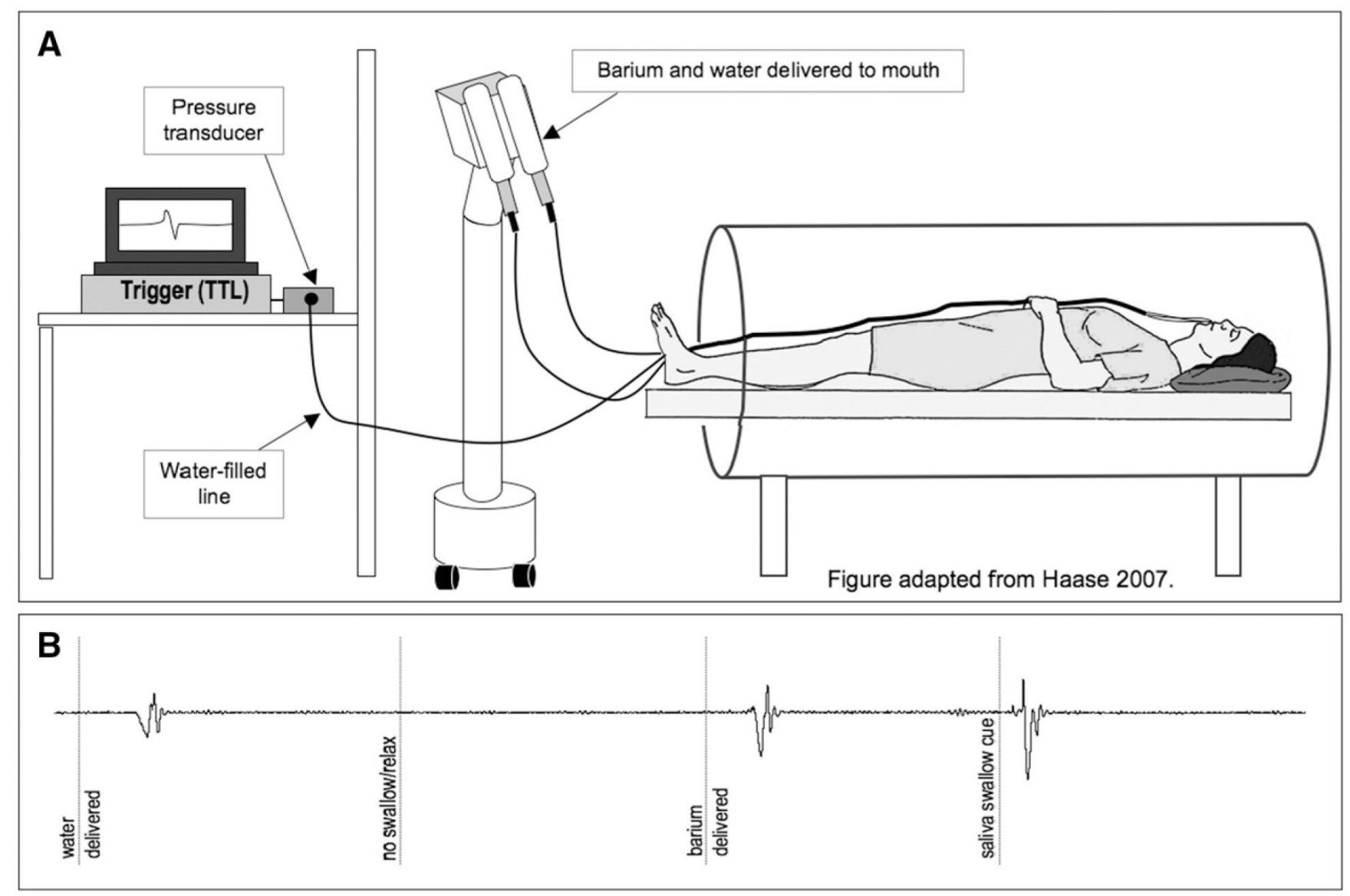

Fig. 1.

fMRI set up and swallow monitoring: A. Bolus delivery and swallow monitoring system: Barium and water are delivered through 2 tubes that extend from programmable syringes. The water-filled line extends from the oral cavity to the pressure transducer to monitor during swallowing activity during scanning. B. Recordings from intra-oral pressure monitoring device during fMRI procedures for each swallowing task. 

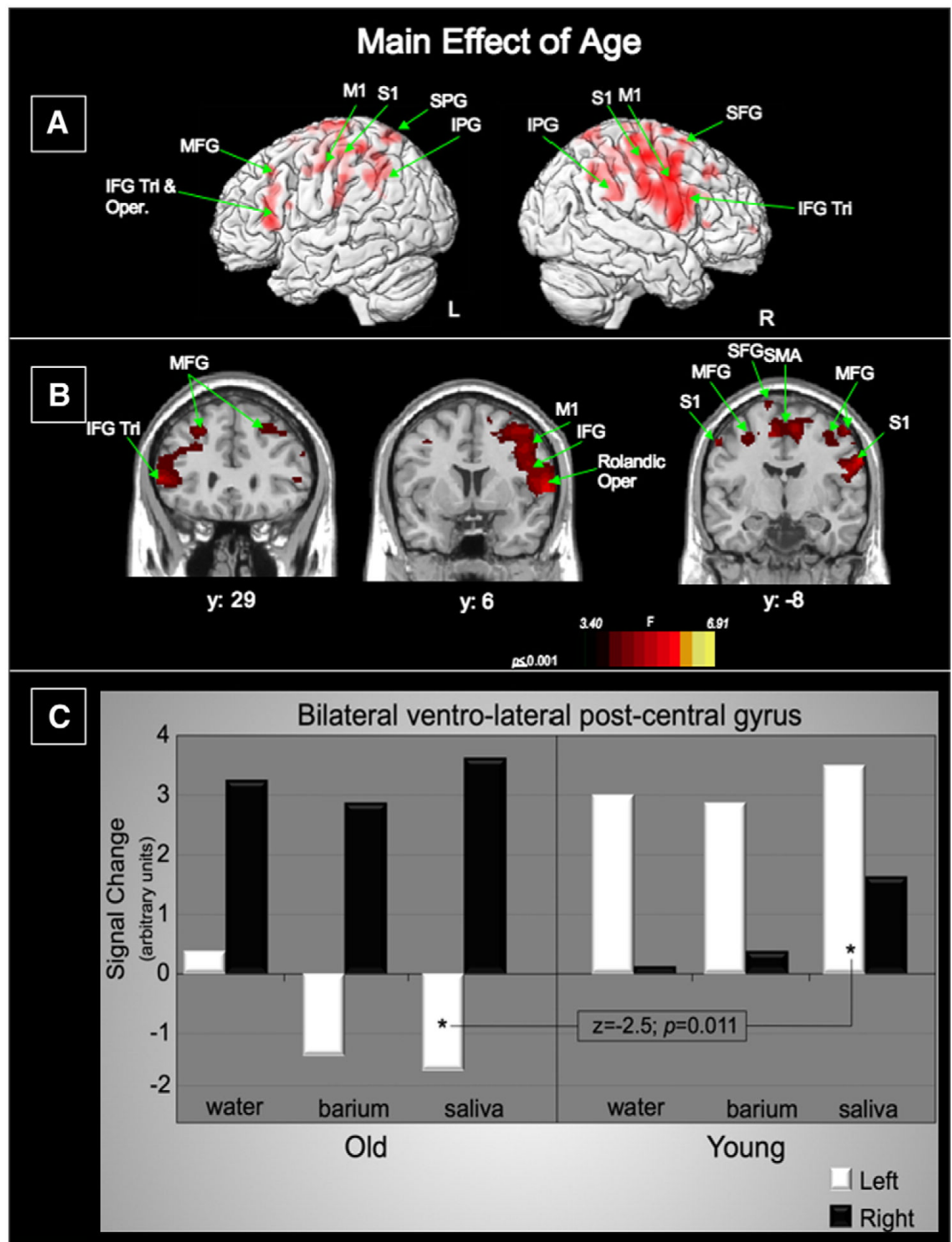

Fig. 2.

Main effect of age: A. Lateral renderings of the brain showing the left and right hemispheres (IFG-inferior frontal gyrus; Tri-triangularis; Oper-opercularis; MFG-middle frontal gyrus; SFG—superior frontal gyrus; M1 - precentral gyrus; S1—postcentral gyrus; IPGinferior parietal gyrus) B. Three coronal slices (IFG—inferior frontal gyrus; Tri-Triangularis; MFG—-middle frontal gyrus; SFG—superior frontal gyrus; M1—precentral gyrus; S1— postcentral gyrus; SMA - supplementary motor area). Left of image is left side of brain. C. Activation in the bilateral ventro-lateral postcentral gyri for each swallow type separated by age group. Within the left ventrolateral postcentral gyrus, only saliva swallows in the old are significantly different from saliva swallows in the young $(z=-2.5 ; p($ corr $)=0.011)$. 


\section{Old > Young}

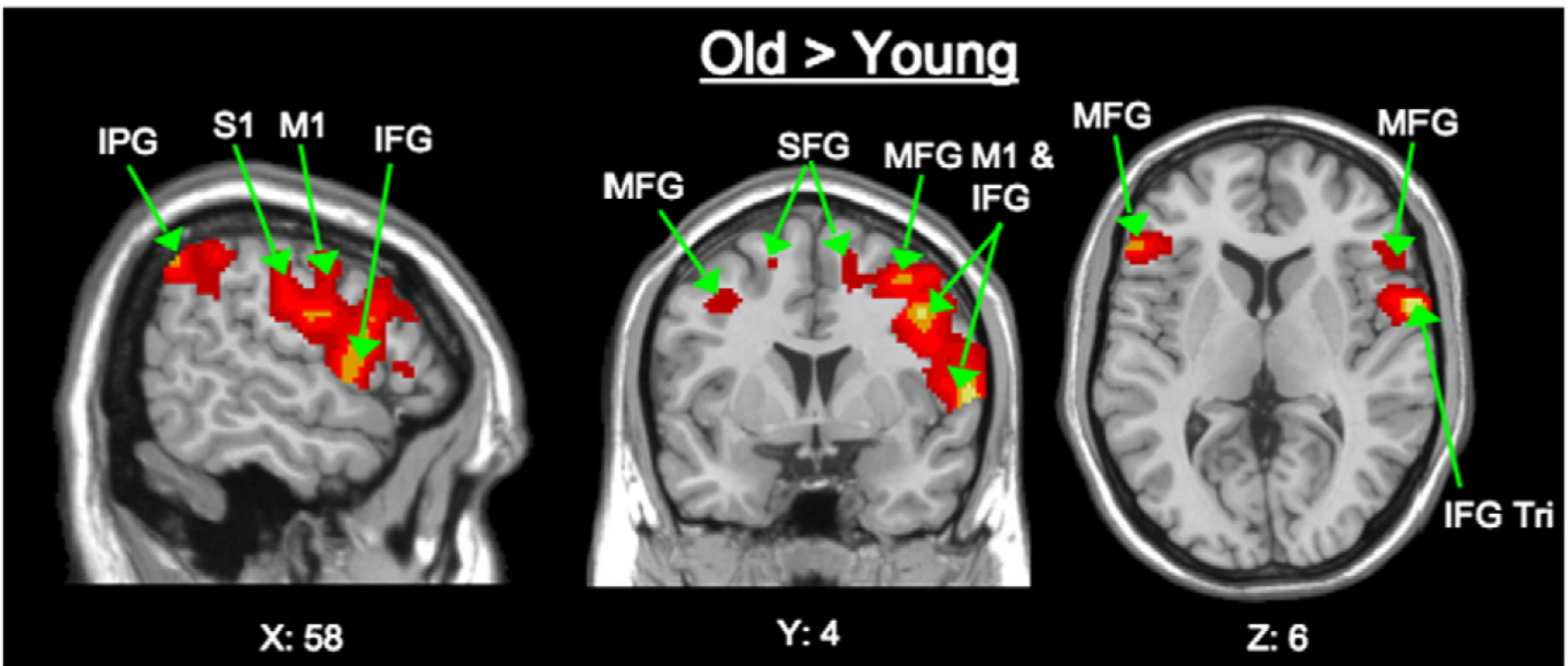

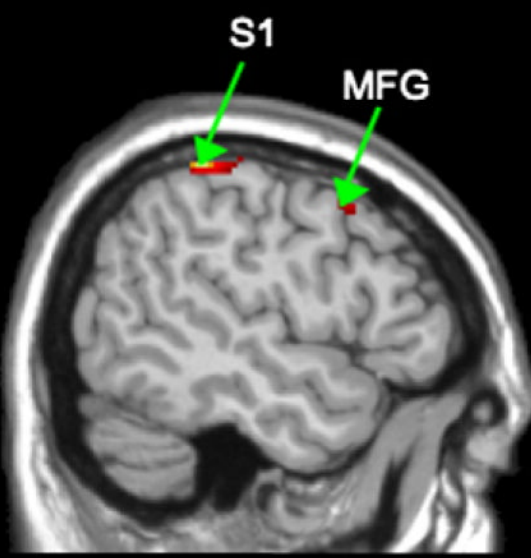

$X:-50$

\section{Young > Old}

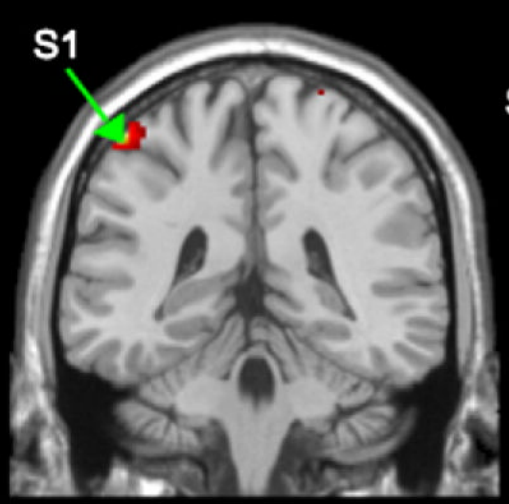

Y: -38

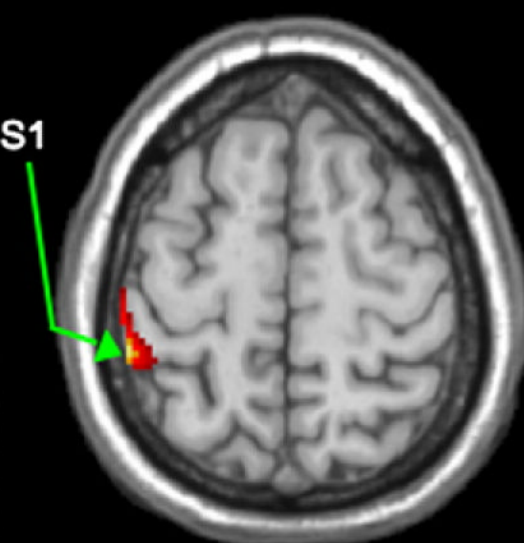

Z: 59

Fig. 3.

Age comparison: Sagital, coronal and axial views of significant BOLD response. Old> Young activation is seen in the right ventro-lateral precentral and postcentral gyri. Young > Old activation is seen in the left precentral and postcentral gyri. Abbreviations: (IFG-inferior frontal gyrus; Tri — triangularis; MFG—-middle frontal gyrus; SFG—-superior frontal gyrus; M1 - precentral gyrus; S1 - postcentral gyrus; IPG — inferior parietal gyrus). Coronal (Y) and axial slices $(\mathrm{Z})$ : Left of image is left side of brain. 

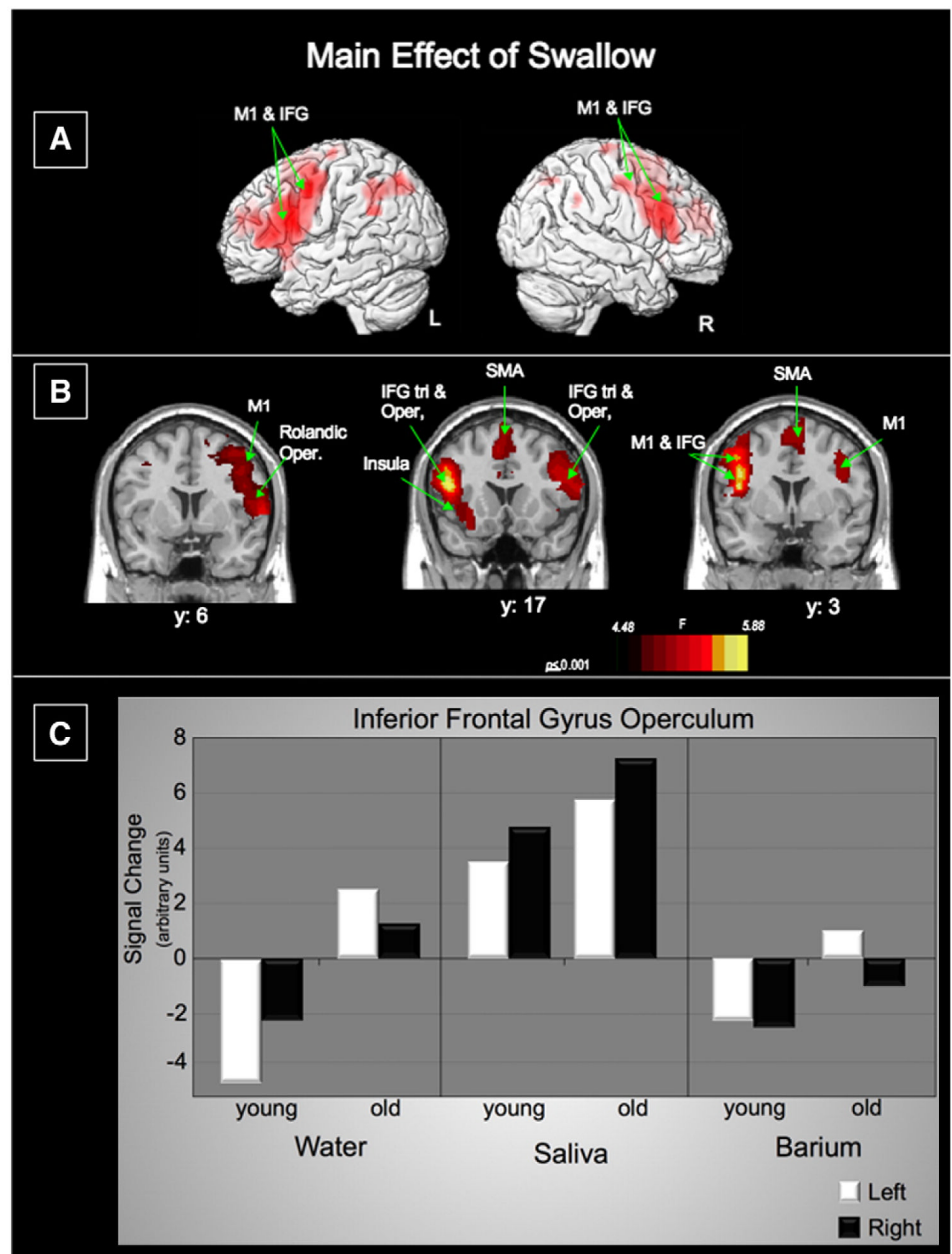

Fig. 4.

Main effect of swallow type: A. Lateral renderings of the brain showing the left and right hemispheres (M1—precentral gyrus; IFG—inferior frontal gyrus) B. Three coronal slices (M1 — precentral gyrus; IFG—inferior frontal gyrus; Tri—triangularis; Oper—opercularis; SMA - supplementary motor area) Left of image is left side of brain. C. Activation in the shown graphically. Activation in the bilateral inferior frontal gyrus pars opercularis for each swallow type separated by age group. 

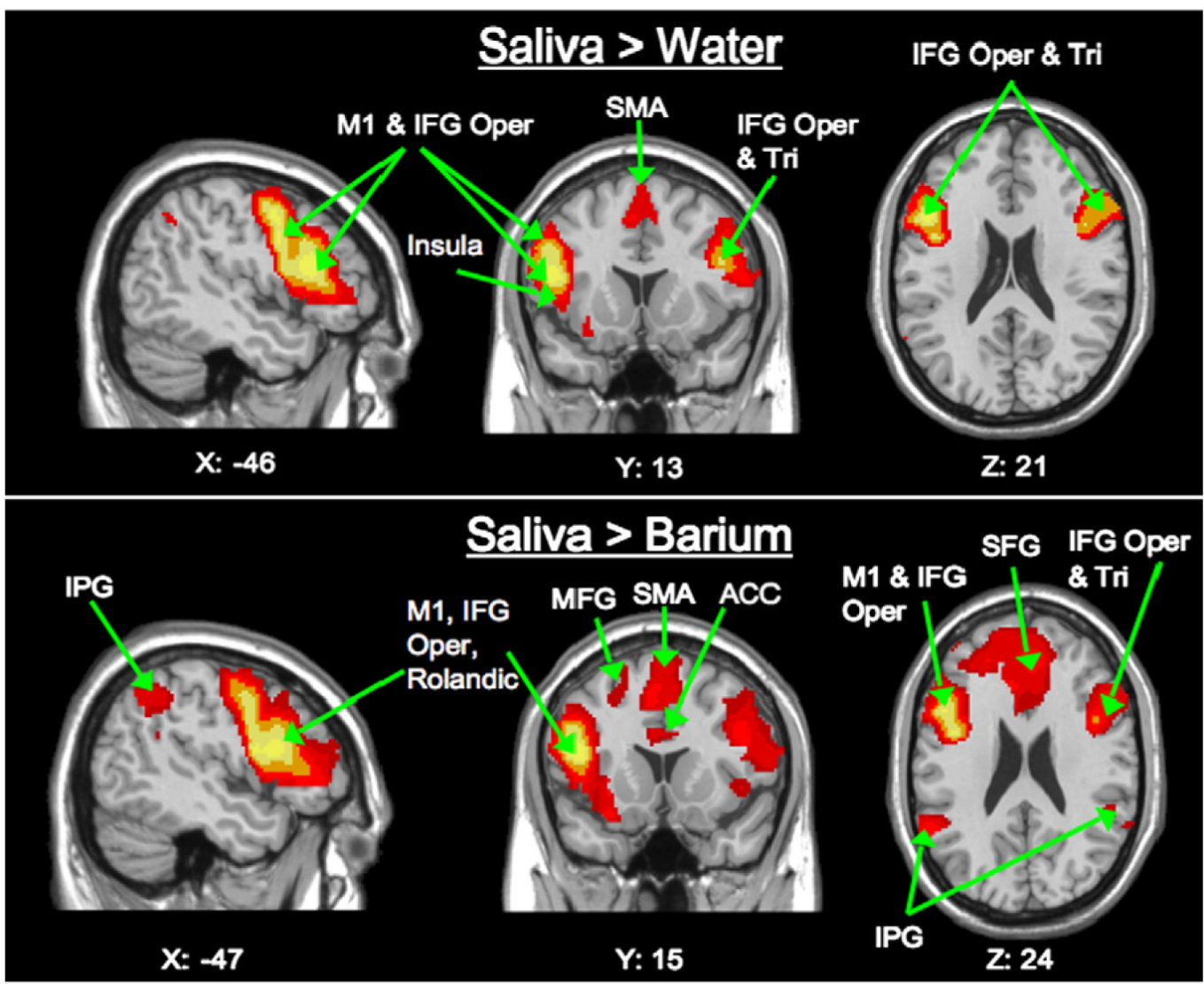

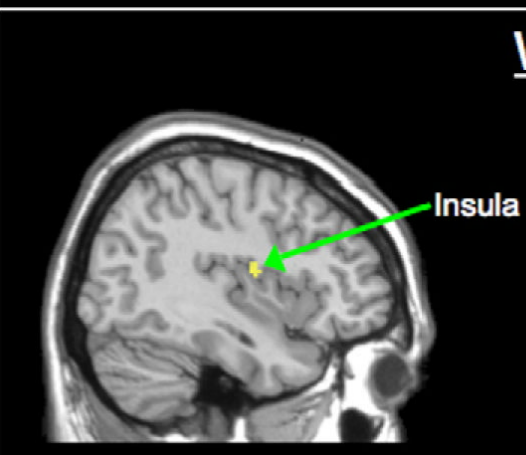

X: 39

\section{Water > Saliva}

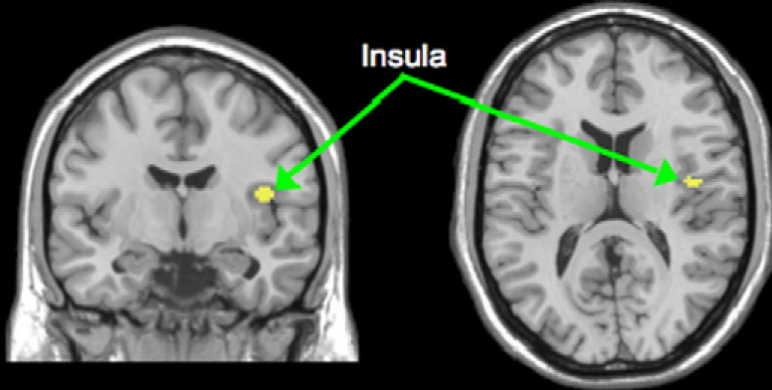

Y: -7

Z: 13

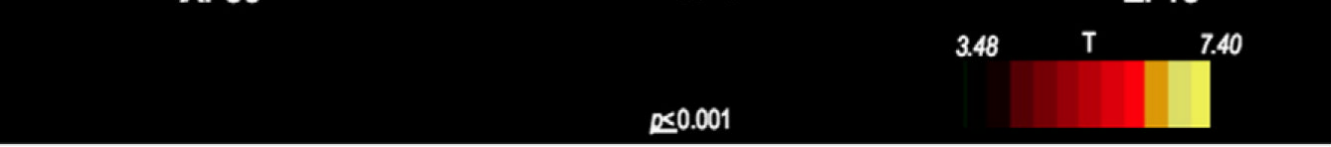

Fig. 5.

Swallow type comparison: Sagittal, coronal and axial views of significant BOLD response. Saliva> Water activation seen in the left ventro-lateral precentral gyrus and bilateral IFG pars opercularis and SMA. Young> Old significant activation is seen in the left precentral gyrus, rolandic and IFG pars opercularis. Water> Saliva activity within the right insular- operculum. Abbreviations: (IFG_-inferior frontal gyrus; Tri-Triangularis; Oper-Opercularis; MFGmiddle frontal gyrus; SFG—superior frontal gyrus; M1—precentral gyrus; IPG—inferior parietal gyrus; ACC-Anterior Cingulate Cortex; SMA — supplementary motor area). Coronal (Y) and axial slices (Z): Left of image is left side of brain. 


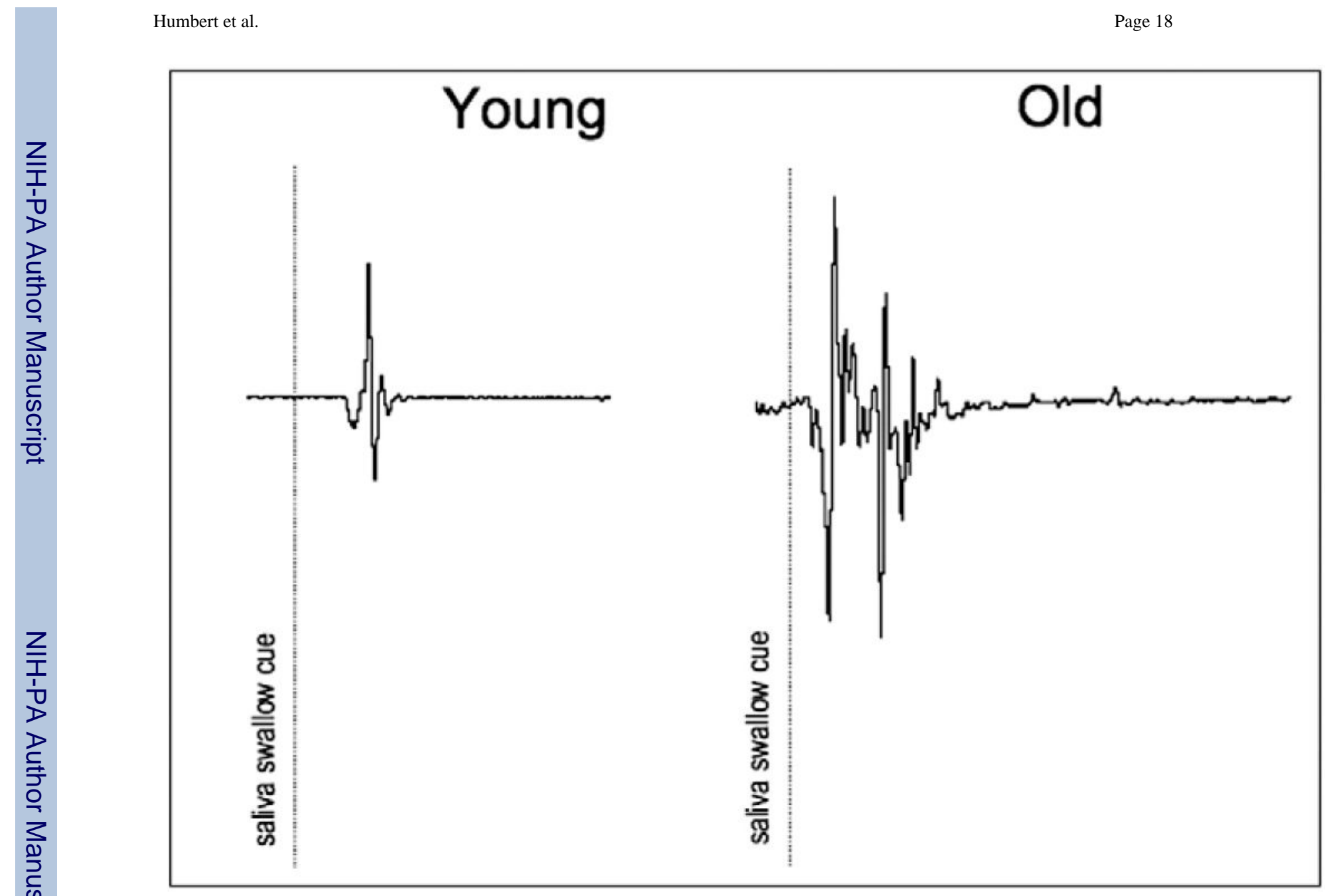

Fig. 6.

Saliva swallow output signals. Representative saliva swallow output signals from the oral pressure monitoring device for and older and younger adult. 


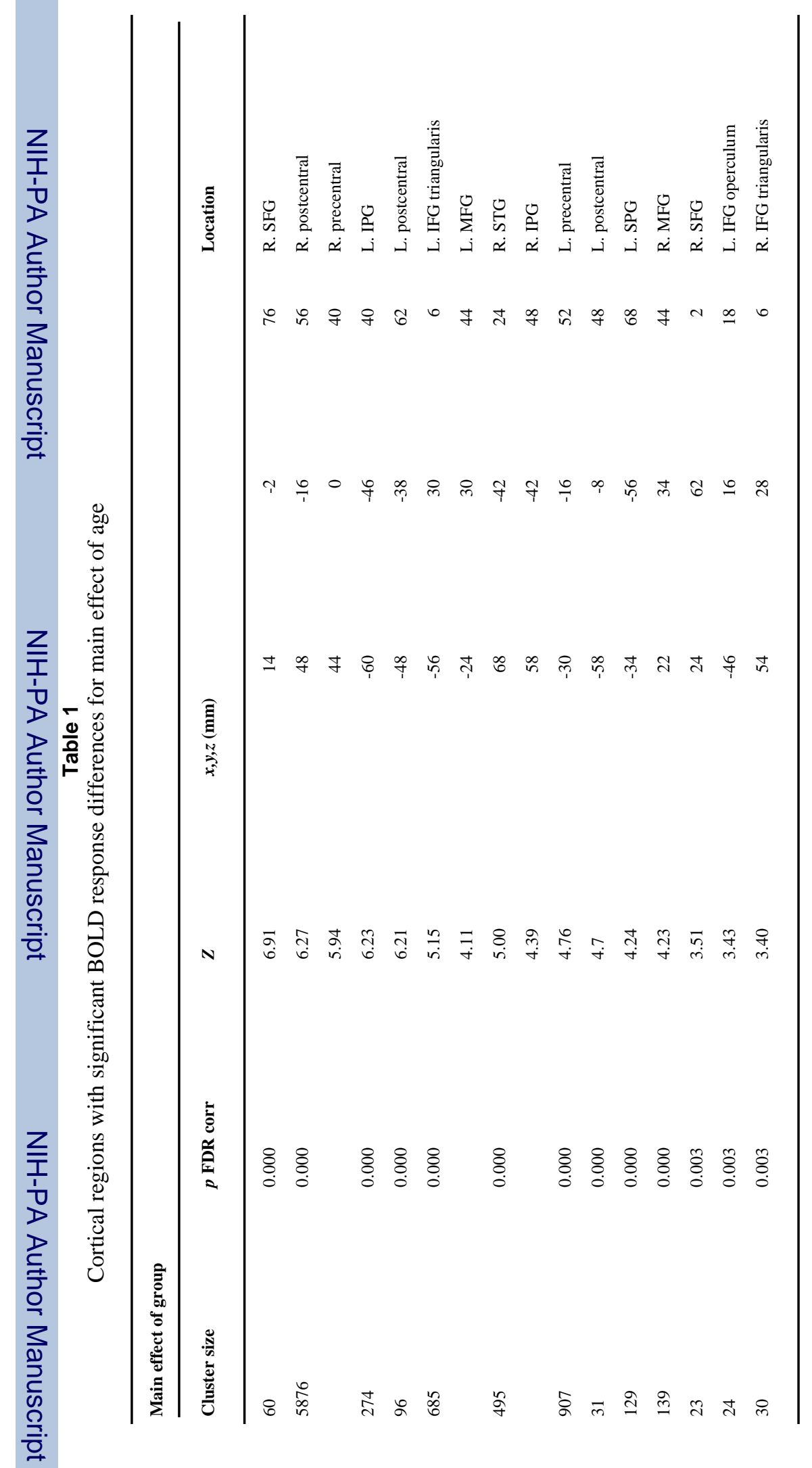




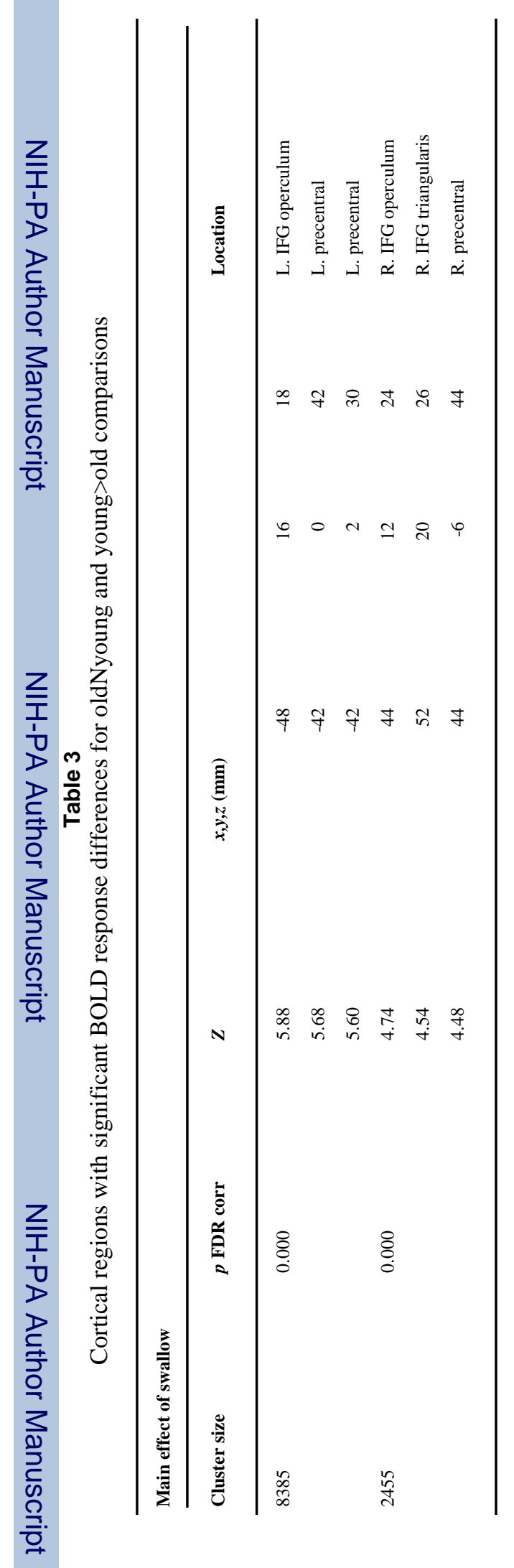




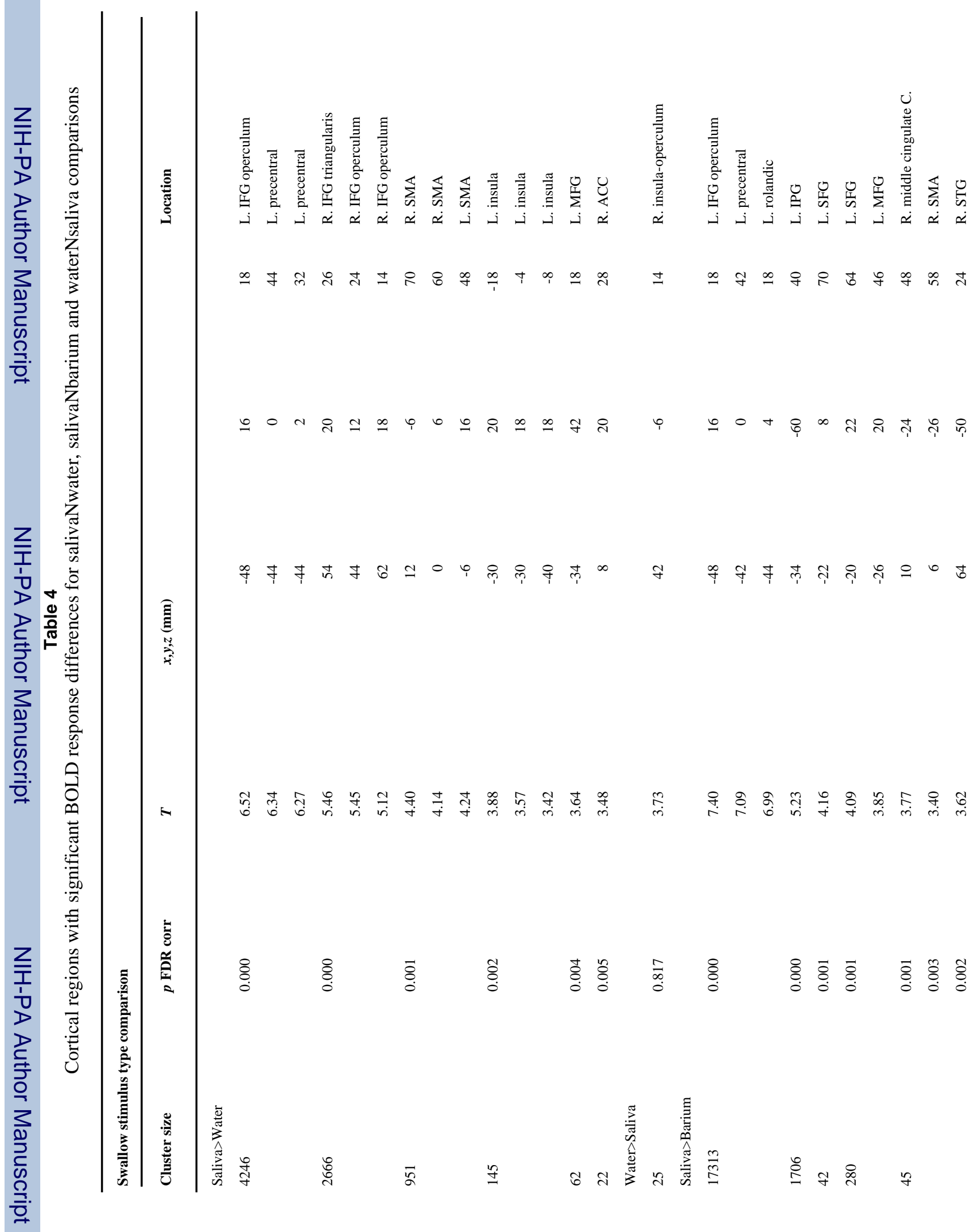




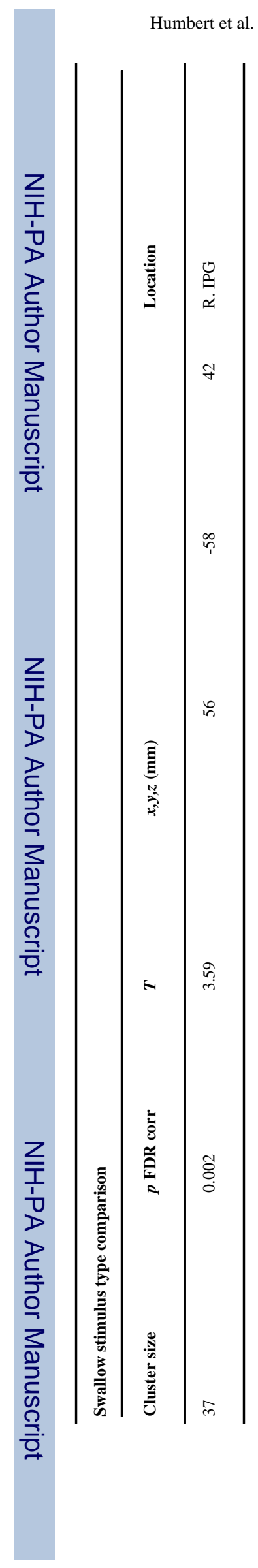

Page 23

Neuroimage. Author manuscript; available in PMC 2010 February 1. 